\title{
Cardiovascular organ damage in type 2 diabetes mellitus: the role of lipids and inflammation
}

Michaela Kozakova ${ }^{1}$, Carmela Morizzo ${ }^{2}$, Isabel Goncalves ${ }^{3}$, Andrea Natali ${ }^{1}$, Jan Nilsson ${ }^{3}$ and Carlo Palombo ${ }^{2^{*}(\text { (D) }}$

\begin{abstract}
Background: The relationship between dyslipidemia, inflammation and CV organ damage in type 2 diabetes mellitus (T2DM) is complex. Insulin resistance and inflammatory cytokines interleukins (ILs) increase plasma triglycerides (TG). ILs also up-regulate expression of matrix-metalloproteinases (MMPs) that, together with TG, decrease high density lipoprotein cholesterol (HDL) levels. High TG, low HDL, increased ILs and MMPs trigger structural and functional changes in different parts of cardiovascular (CV) system. To understand better the role of lipids and inflammation in CV organ damage, the present study investigated the inter-relationships between lipids, ILs and MMPs, as well as the associations of lipids, ILs and MMPs with various CV measures, both in diabetic and non-diabetic population (nonT2DM).
\end{abstract}

Methods: In T2DM patients ( $N=191)$ and nonT2DM subjects $(N=94)$ were assessed carotid intima-media thickness (cIMT) and inter-adventitial diameter (IADiam), carotid wave speed (ccaWS), carotid-femoral pulse wave velocity (cfPWV), left ventricular (LV) mass, LV systolic (s') and early diastolic (e') longitudinal velocities of mitral annulus, together with glycemic control, lipid profile, IL-6, IL-18 and MMP-12.

Results: T2DM patients, as compared to nonT2DM subjects, had significantly higher plasma levels of IL-6, IL-18, MMP-12 and lower HDL ( $P<0.05-0.0001)$. They had also higher cIMT, IADiam, ccaWS, cfPWV and LV mass, and lower $\mathrm{e}^{\prime}$ velocity $(P<0.005-0.0001)$. Both in T2DM patients and nonT2DM subjects, MMP-12 increased with IL-6 $(r=0.43$ and $0.39 ; P<0.0001)$ and IL-18 ( $r=0.32$ and $0.42 ; P<0.0001)$, and HDL decreased with MMP-12 ( $r=-0.29$ and -0.42 ; $P<0.0001)$. In both populations, MMP-12 was directly associated with IADiam, ccaWS, cfPWV and LV mass ( $r=0.42$, $0.32,0.26$ and $0.29 ; P<0.0001$ in T2DM patients, and $r=0.39,0.28,0.32$ and $0.27 ; P<0.01-0.0001$ in nonT2DM subjects). In multivariate analysis, MMP-12 remained independently related to IADiam, ccaWS, cfPWV and LV mass in T2DM patients, and to IADiam only in nonT2DM subjects.

Conclusions: This cross-sectional study demonstrated a direct association between ILs and MMP-12, as well as an inverse association between MMP-12 and HDL, both in T2DM patients and in nonT2DM subjects. In T2DM patients, who had higher levels of ILs and MMP-12, the latter was independently related to several structural and functional markers of preclinical CV organ damage.

Keywords: Diabetes mellitus, Interleukins, Matrix-metalloproteinase, High density lipoprotein cholesterol, Arterial stiffness, Left ventricular mass

\footnotetext{
*Correspondence: carlo.palombo@unipi.it

${ }^{2}$ Department of Surgical, Medical Molecular Pathology and Critical Care

Medicine, University of Pisa, Via Savi 10, 56126 Pisa, Italy

Full list of author information is available at the end of the article
} 


\section{Background}

Type 2 diabetes mellitus (T2DM) is associated with increased cardiovascular (CV) morbidity and mortality [1] and a major pathophysiologic mechanism linking T2DM to CV risk is diabetic dyslipidaemia, characterized by high triglycerides (TG) and low high density lipoprotein cholesterol (HDL) plasma levels [2]. The two lipids are closely connected in T2DM, since the insulin resistance-induced increase in plasma TG triggers the catabolism of HDL, and consequently, a specific role of the two lipids in CV organ damage is not clearly understood [3]. Moreover, T2DM is a chronic low-grade inflammation state [4], and inflammatory cytokines may also influence plasma lipids levels $[5,6]$. Interleukins (ILs) stimulate hepatic secretion of TG [6] and up-regulate expression and activity of matrix-metalloproteinases (MMPs) [7], the endopeptidases that decrease HDL levels through degradation of apolipoprotein A-I [8]. Yet, interleukins and MMPs may not only modify lipid profile but also directly damage CV system [9-13].

It is evident that the relationship between lipids, inflammation and $\mathrm{CV}$ organ damage in T2DM patients is complex. Number of studies have shown the associations of TG, HDL, ILs or MMPs with vascular and cardiac alterations [9, 12-17], yet the majority of these studies have evaluated the impact of a single metabolic abnormality on a single CV measure, without taking into consideration the relationships between lipids and inflammation and without considering that the heart and large arteries are closely anatomically and functionally linked and that the changes in vascular tree have impact on the structure and function of the heart.

To understand better the role of dyslipidemia and inflammation in CV organ damage and CV risk of T2DM patients, the present study evaluated the inter-relationships between TG, HDL, ILs and MMP-12, as well as the associations of lipids, ILs and MMP-12 with different measures of $\mathrm{CV}$ structure and function, both in T2DM patients and in subjects free of diabetes (nonT2DM).

\section{Methods}

\section{Study population}

Study population consists of 191 T2DM patients and 94 nonT2DM subjects of similar age and comparable prevalence of CV disease. All subjects were referred for a complete CV examination to the Clinic for Cardiometabolic Risk Prevention of the Department of Surgical and Medical Pathology, University of Pisa between December 2010 and April 2013. Diagnosis of T2DM was based on plasma glucose criteria (fasting glucose $\geq 7 \mathrm{mmol} / \mathrm{L}$ or 2 -h glucose during oral-glucose tolerance test $\geq 11.1 \mathrm{mmol} / \mathrm{L}$ ) or $\mathrm{HbA} 1 \mathrm{c} \geq 6.5 \%$ [18]. Two abnormal test results from the same sample or in two separate test samples were required to confirm the diagnosis.

Twenty-one T2DM patients were treated by diet, 135 by oral antidiabetic drugs, 22 by a combination of oral antidiabetics and insulin and 13 by insulin only. Antagonists of the renin-angiotensin-aldosterone system (ACE inhibitors and ARBs) were the most frequently used antihypertensive agent, followed by beta-blockers, both in T2DM patients and nonT2DM subjects.

\section{Study protocol}

The protocol of the study followed the principles of the Declaration of Helsinki and was approved by the institutional ethics committee "Comitato Etico di Area Vasta Nord Ovest” (Reference Number: 3146/2010). All subjects gave their informed consent to participate.

\section{Vascular examination}

All study subjects underwent carotid ultrasound and measurement of carotid-femoral pulse wave velocity (cfPWV). Vascular examination was performed in the afternoon, $3 \mathrm{~h}$ after a light meal, in a quiet room with a stable temperature of $22^{\circ}$, after resting comfortably for at least $15 \mathrm{~min}$ in the supine position. All subjects were asked to abstain from cigarette smoking, caffeine and alcohol consumption and vigorous physical activity for $24 \mathrm{~h}$.

CfPWV was measured according to current guidelines using the Complior device (Alam Medical, Vincennes, France). In our laboratory, intra- and inter-individual variability of cfPWV measurement are $4.3 \pm 2.8 \%$ and $5.1 \pm 2.9 \%$, respectively. Increase in cfPWV was defined as cfPWV higher than 90th age- and BP-specific percentiles of cfPWV observed in a reference population of 11.092 subjects [19].

Carotid ultrasound was performed by a single operator $(\mathrm{CM})$ on the right common carotid artery using an ultrasound scanner equipped with a $10 \mathrm{MHz}$ linear probe (MyLab 70, Esaote, Genova, Italy) and implemented with a previously validated radiofrequency-based tracking of arterial wall (QIMT ${ }^{\circledR}$ and QAS $^{\circledR}$ ) that allows an automatic and real-time determination of far-wall cIMT, IADiam and carotid distension with a high spatial and temporal resolution (sampling rate of $550 \mathrm{~Hz}$ on 32 lines). From the distension curves a local one-point carotid wave speed (ccaWS) was calculated applying the Bramwell-Hill equation that relates the propagation velocity to arterial distensibility as previously described [20]. Increase in cIMT was defined as IMT higher than 90th age- and sex-specific percentiles of cIMT observed in a reference population of 1.993 men and women [21]. All radiofrequency-derived measures were averaged over 6 consecutive cardiac beats and the values used for 
statistical analysis represent a mean of three consecutive acquisitions.

\section{Echocardiographic examination}

Left ventricle (LV) mass was measured by echocardiography (MyLab 70, Esaote, Genova, Italy, equipped with a 1.8-3.5 MHz, phased-array probe). LV inner diameter and wall thickness were measured in end-diastole and end-systole in M-mode images, and LV mass was calculated by Penn formula [22]. LV mass index was calculated as LV mass per height ${ }^{2.7}$ and LV hypertrophy was defined as LV mass index $>51 \mathrm{~g} / \mathrm{m}^{2.7}$ in both men and women [23]. End-systolic wall stress was calculated as recommended [24]. Stroke volume was assessed as the product of aortic valve cross-sectional area and transaortic flowvelocity time integral.

Systolic and diastolic LV longitudinal velocities at mitral annular level, both at septal and lateral sides, were measured by color-guided pulsed-wave tissue Doppler in the apical four-chamber view. The sample volume was placed at the junction of the LV wall with the mitral annulus, and the cursor was aligned so that the angle of incidence between the Doppler beam and the longitudinal motion of the $L V$ was as close as possible to $0^{\circ}$. From spectral traces, peak longitudinal velocity during systole $\left(\mathrm{s}^{\prime}\right)$ and during early diastolic filling $\left(\mathrm{e}^{\prime}\right)$ were measured and averaged over 5 consecutive cardiac cycles [25]. Reported values represent an average of septal and lateral sides. The intra-individual variability of tissue Doppler measurements in our laboratory is $4.7 \pm 3.5 \%$ and $5.8 \pm 4.3 \%$ for $\mathrm{s}^{\prime}$ and $\mathrm{e}^{\prime}$ velocity, respectively.

\section{Medical history, physical and instrumental examination}

A standardized medical history, physical examination and resting ECG and echocardiography were performed in all subjects. CV disease was defined as coronary artery disease and unstable angina, infarction, heart failure, stroke or peripheral macrovascular disease. Height and weight were obtained and body mass index (BMI) was calculated. Waist circumference was measured as the narrowest circumference between the lower rib margin and anterior superior iliac crest. Office brachial BP was measured twice during two different visits in a seated patient, using a standard mercury sphygmomanometer; regular or large adult cuffs were used, depending on patient arm circumference. The mean value of the two measurements was calculated and used for statistical analysis. Smoking habit was evaluated as never, past and current smoker.

\section{Analytical procedures}

Plasma levels of low density lipoprotein cholesterol (LDL), HDL, TG and glucose were determined within
1 week of CV examination by standard methods on a Roche-Modular Autoanalyzer (Milan, Italy). Glycosylated hemoglobin (HbA1c) was measured by high-performance liquid chromatography and standardized against Diabetes Control and Complications Trial (DCCT ) standard. Plasma levels of biomarkers reflecting inflammation (IL-6 and IL-18) and extracellular matrix proteolysis (MMP12) were analyzed by the Proximity Extension Assay (PEA) technique using the Proseek Multiplex CVD ${ }^{96 \times 96}$ reagents kit (Olink Bioscience, Uppsala, Sweden) at the Clinical Biomarkers Facility, Science for Life Laboratory, Uppsala as previously described $[9,26]$. All samples were analyzed in the same run. Data analysis was performed by a preprocessing normalization procedure using Olink Wizard for GenEx (Multid Analyses, Sweden). Values are presented as arbitrary units (AU). Data regarding intraand inter-assays variations as well as general calibrator curves to calculate the approximate concentrations are available on the OLINK homepage (http://www.olink .com).

\section{Statistical analysis}

Data are expressed as mean $\pm \mathrm{SD}$, categorical data as percentages. Variables with skewed distribution were summarized as median [interquartile range], and were logarithmically transformed for parametric statistical analysis. ANOVA was used to compare continuous variables, and a $X^{2}$ test for categorical variables. The univariate relationships between the outcome variables and continuous variables were assessed by Pearson (r) correlation coefficient. Multiple linear regression analyses (controlled for age, sex, body size, BP, heart rate, smoking habit, BP-lowering and lipid-lowering therapy and, in T2DM patient, also for diabetes duration and antidiabetic treatment) with backward stepwise removal were used to identify the independent associations of outcome variables with their significant univariate correlates. Statistical tests were two-sided, and significance was set at a value of $P<0.05$. Statistical analysis was performed by JMP software, version 3.1 (SAS Institute Inc., Cary, NC, USA).

\section{Results}

\section{Characteristics of the study population}

Characteristics of T2DM patients and nonT2DM subjects are reported in Table 1. As compared to nonT2DM subjects, T2DM patients were on average 3 years older and had significantly higher body size, systolic BP and heart rate, plasma levels of fasting glucose, Hb1AC, ILs and MMP-12, and lower plasma HDL levels. The prevalence of BP-lowering and lipidlowering therapy, but not that of $\mathrm{CV}$ events, was higher in T2DM. T2DM patients had higher cIMT, IADiam, 
Table 1 Characteristics of study population (mean \pm SD/median [IQR])

\begin{tabular}{|c|c|c|c|}
\hline & T2DM patients & nonT2DM subjects & \\
\hline Gender (M:F) & $148: 43$ & $66: 28$ & 0.20 \\
\hline Age (years) & $65 \pm 7$ & $62 \pm 8$ & $<0.0001$ \\
\hline T2DM duration (years) & $6[11]$ & & \\
\hline Body weight (kg) & $84 \pm 15$ & $79 \pm 12$ & $<0.005$ \\
\hline $\mathrm{BMI}\left(\mathrm{kg} / \mathrm{m}^{2}\right)$ & $29.0 \pm 4.5$ & $27.2 \pm 3.6$ & $<0.001$ \\
\hline Waist circumference (cm) & $106 \pm 12$ & $98 \pm 11$ & $<0.0001$ \\
\hline Systolic BP/diastolic BP (mmHg) & $135 \pm 19 / 77 \pm 10$ & $128 \pm 15 / 78 \pm 10$ & $<0.05^{\mathrm{a}}$ \\
\hline Heart rate (bpm) & $66 \pm 11$ & $60 \pm 9$ & $<0.0001$ \\
\hline LDL-cholesterol (mmol/L) & $2.67 \pm 0.85$ & $2.86 \pm 0.94$ & 0.09 \\
\hline HDL-cholesterol (mmol/L) & $1.27 \pm 0.28$ & $1.35 \pm 0.35$ & $<0.05$ \\
\hline Triglycerides (mmol/L) & $1.32[0.82]$ & $1.25[0.61]$ & 0.08 \\
\hline Fasting glucose (mmol/L) & $7.4 \pm 1.8$ & $4.9 \pm 0.6$ & $<0.0001$ \\
\hline $\mathrm{HbA1c}(\%)$ & $7.0[1.4]$ & $5.7[0.4]$ & $<0.0001$ \\
\hline $\operatorname{hsCRP}(\mathrm{mg} / \mathrm{dL})$ & $0.15[0.26]$ & $0.17[0.19]$ & 0.40 \\
\hline IL-6 (AU) & $36[25]$ & $29[25]$ & $<0.01$ \\
\hline IL-18 (AU) & $1314[637]$ & 1140 [557] & 0.005 \\
\hline MMP-12 (AU) & $139[96]$ & $98[58]$ & $<0.0001$ \\
\hline BP-lowering therapy (\%) & 73.3 & 52.1 & $<0.0005$ \\
\hline Lipid-lowering therapy (\%) & 62.8 & 50.0 & $<0.05$ \\
\hline Current smoking (\%) & 31.1 & 34.0 & 0.57 \\
\hline CV events (\%) & 49.2 & 40.4 & $0.44^{\mathrm{a}}$ \\
\hline $\mathrm{cIMT}(\mu \mathrm{m})$ & $782 \pm 129$ & $668 \pm 139$ & $<0.01^{\mathrm{a}}$ \\
\hline cIMT > 90th percentile (\%) & 33.5 & 18.1 & 0.005 \\
\hline IADiam (mm) & $8.52 \pm 0.91$ & $8.05 \pm 0.92$ & $<0.005^{\mathrm{a}}$ \\
\hline $\operatorname{ccaWS}(\mathrm{m} / \mathrm{s})$ & $8.9 \pm 1.8$ & $7.9 \pm 1.5$ & $0.001^{\mathrm{a}}$ \\
\hline $\operatorname{cfPWV}(\mathrm{m} / \mathrm{s})$ & $11.4 \pm 2.4$ & $9.3 \pm 2.0$ & $<0.0001^{\mathrm{a}}$ \\
\hline cfPWV > 90th percentile (\%) & 27.2 & 4.3 & $<0.0001$ \\
\hline LV mass $(\mathrm{g})$ & $225 \pm 54$ & $199 \pm 52$ & $<0.0005$ \\
\hline LV hypertrophy (\%) & 51.8 & 29.8 & $<0.001$ \\
\hline $\mathrm{s}^{\prime}$ velocity $(\mathrm{cm} / \mathrm{s})$ & $8.6 \pm 1.3$ & $8.8 \pm 1.6$ & 0.28 \\
\hline $\mathrm{e}^{\prime}$ velocity $(\mathrm{cm} / \mathrm{s})$ & $8.7 \pm 1.4$ & $9.7 \pm 2.0$ & $<0.0005^{\mathrm{a}}$ \\
\hline Stroke volume $(\mathrm{mL})$ & $81 \pm 16$ & $70 \pm 19$ & $<0.0001$ \\
\hline End-systolic wall stress $\left({ }^{*} 10^{3}\right.$ dynes $\left./ \mathrm{cm}^{2}\right)$ & $72 \pm 24$ & $69 \pm 29$ & 0.44 \\
\hline
\end{tabular}

a After adjustment for age

ccaWS, cfPWV, LV mass and lower e' velocity. The prevalence of established organ damage (increased cIMT, cfPWV and LV hypertrophy) was also higher in T2DM.

Inter-relationships between lipids, ILs and MMP-12 In both T2DM patients (Fig. 1) and nonT2DM subjects (Fig. 2), HDL decreased with TG and MMP-12, and MMP-12 increased with IL-6 and IL-18. In T2DM only, TG increased with fasting plasma glucose levels $(\mathrm{r}=0.35 ; P<0.0001)$. Fasting glucose and HbA1c was not related to ILs in either population.
CV measures, lipids, ILs and MMP-12

In T2DM patients, cIMT, IADiam, ccaWS, cfPWV and LV mass were inversely related to HDL $(r=-0.31$, $-0.26,-0.24,-0.21$ and $-0.23 ; P<0.005-0.0001$, and IADiam, ccaWS, cfPWV and LV mass were directly related to MMP-12 (Fig. 3). LV mass was also directly related to fasting glucose and IL- $6(\mathrm{r}=0.20$ and 0.25 ; $P<0.01$ and $<0.001)$. E' velocity of mitral annulus was inversely related to fasting glucose $(\mathrm{r}=-0.18 ; P<0.05)$ and directly to HDL $(\mathrm{r}=0.24 ; P<0.001)$, whereas $\mathrm{s}^{\prime}$ velocity was not related to any metabolic parameter.

In nonT2DM subjects, LV mass was related inversely $(\mathrm{r}=-0.37 ; P<0.0005)$ and $\mathrm{e}^{\prime}$ and $\mathrm{s}^{\prime}$ velocities were 

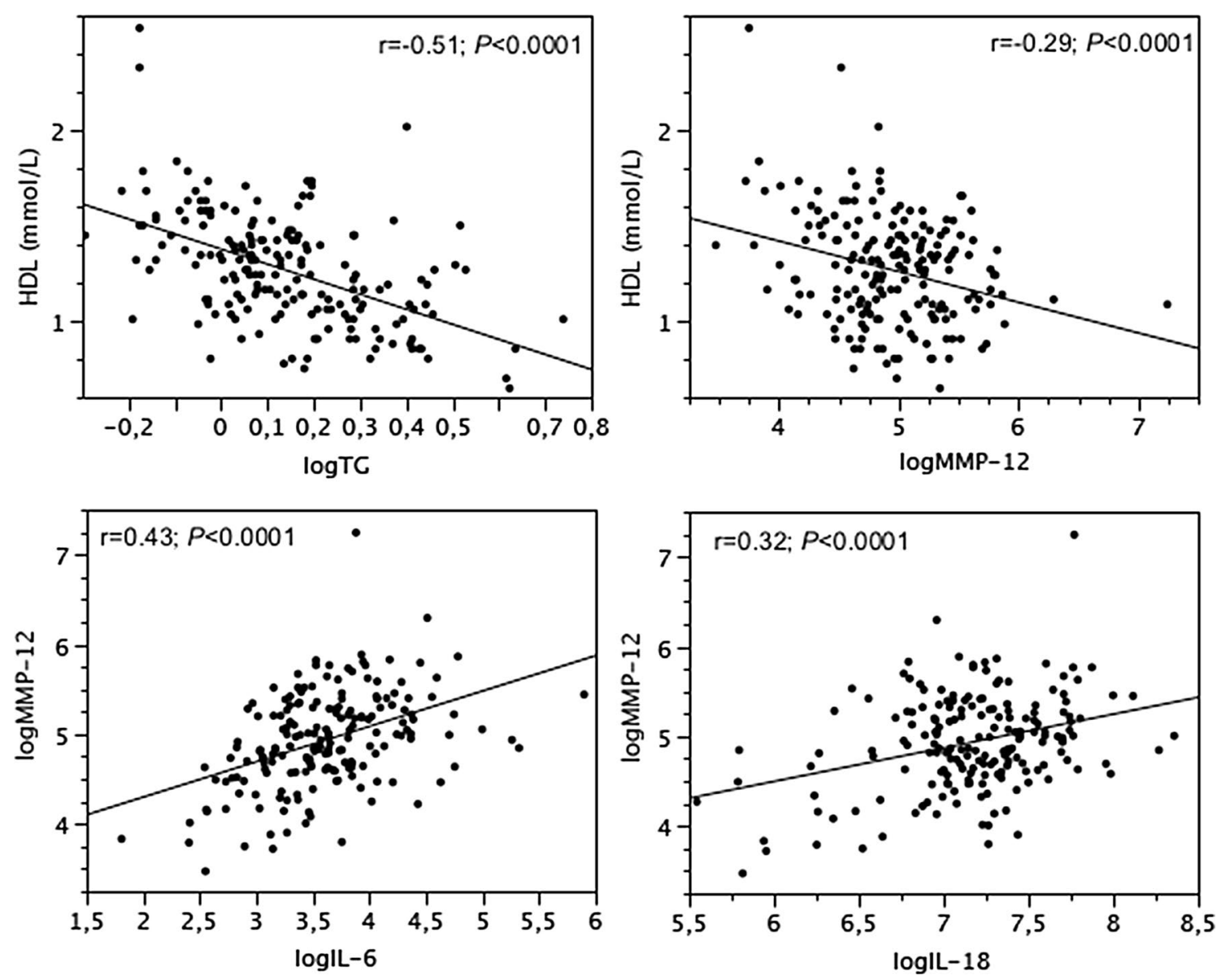

Fig. 1 Relationships between HDL, TG and MMP-12, and relationships between MMP-12, IL-6 and IL-18 in T2DM patients

related directly to HDL $(\mathrm{r}=0.34$ and $0.26 ; P=0.001$ and 0.01). IADiam, ccaWS, cfPWV and LV mass were directly related to MMP-12 (Fig. 4). CfPWV was also related to fasting glucose $(\mathrm{r}=0.22 ; P<0.01)$.

In both T2DM patients and nonT2DM subjects, cfPWV was directly related to end-systolic LV wall stress $(\mathrm{r}=0.32$ and $0.26 ; P<0.0001$ and $<0.01)$, and $\mathrm{s}^{\prime}(\mathrm{r}=-0.25$ and $-0.27 ; P<0.0005$ and $<0.01)$ and $\mathrm{e}^{\prime}(\mathrm{r}=-0.23$ and $-0.32 ; P<0.005$ for both) velocities were inversely related to LV mass. Only in T2DM patients, cfPWV was directly related to $L V$ mass $(r=0.26 ; P<0.0005)$ and $s^{\prime}$ velocity was inversely related to end-systolic wall stress $(\mathrm{r}=-0.23 ; P=0.001)$.

The independence of associations between glucose, lipids, ILs, MMP-12 and vascular (Table 2) and cardiac (Table 3) measures was tested in multiple regression models with stepwise removal, after adjustment for age, sex, body size, BP, heart rate, smoking habit, BP-lowering and lipid-lowering therapy and, in T2DM patients, also for diabetes duration and antidiabetic treatment. In T2DM patients, HDL was independently related to cIMT and $\mathrm{e}^{\prime}$ diastolic velocity and MMP-12 was independently related to IADiam, ccaWS, cfPWV and LV mass. $S^{\prime}$ velocity decreased with peak systolic wall stress and LV mass. Plasma glucose, HbA1c or TG were not independently related to $\mathrm{CV}$ measures (Tables 2 and 3). In nonT2DM subjects, CV measures were predominantly determined by age, sex and hemodynamic parameters; only IADiam was related to MMP-12 and $\mathrm{s}^{\prime}$ and $\mathrm{e}^{\prime}$ velocities were related to HDL (Tables 2 and 3).

\section{$\mathrm{CV}$ organ damage and $\mathrm{CV}$ disease}

T2DM patients with CV organ damage, i.e. those with CIMT and cfPWV higher than 90th percentiles of the reference population and those with the presence of LV hypertrophy, had significantly lower HDL and higher ILs and MMP-12 than those without organ damage (Table 4), after adjustment for diabetes duration, BPlowering, lipid-lowering and diabetic treatment, smoking habit and, if applicable, also for sex, age and BP. The two subgroups did not differ for fasting glucose, HbA1c and TG. T2DM patients with CV disease had, as compared 

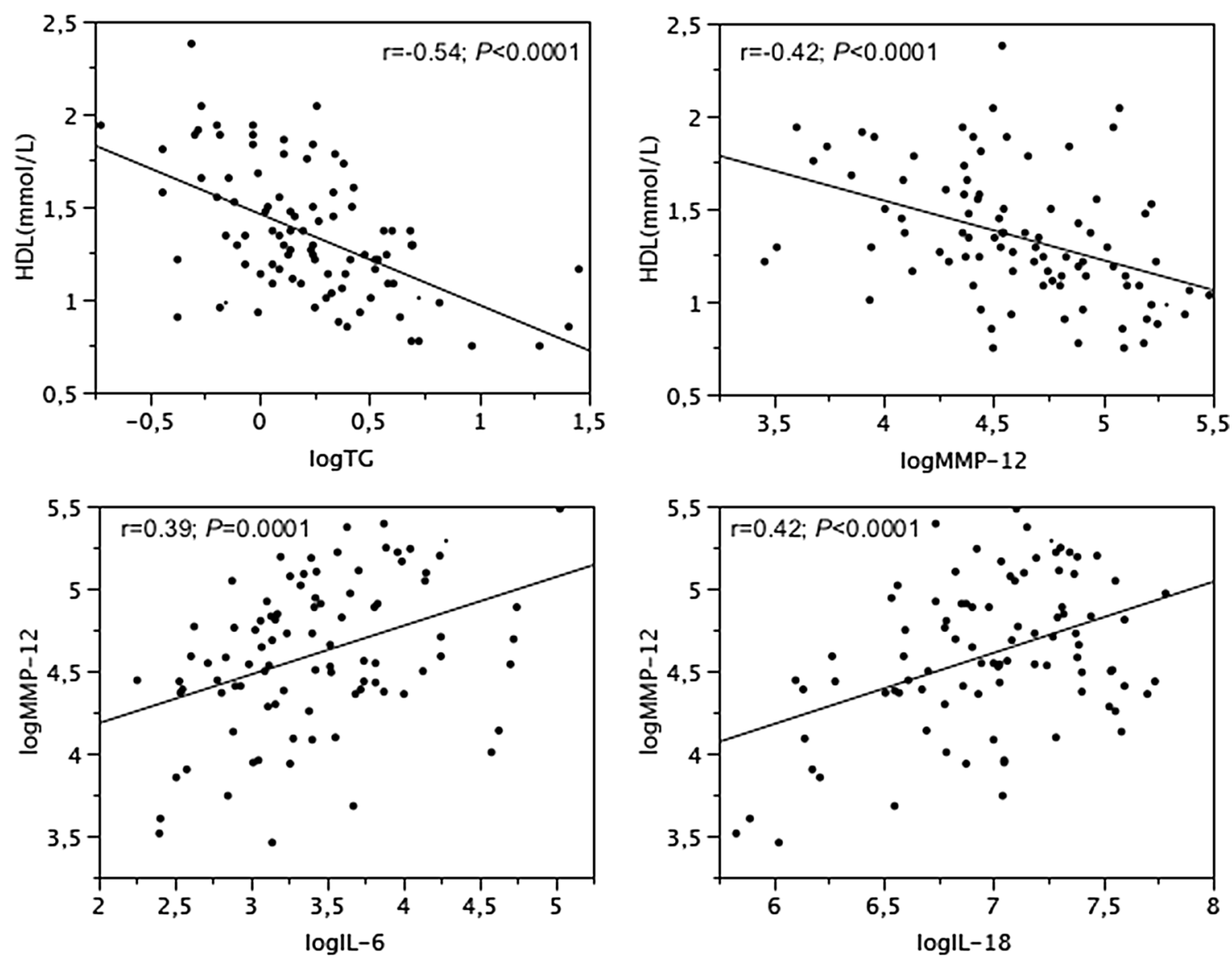

Fig. 2 Relationships between HDL, TG and MMP-12, and relationships between MMP-12, IL-6 and IL-18 in nonT2DM subjects

to those without CV disease, higher IL-6 and cfPWV (Table 4), after adjustment for sex, age, BP, smoking habit, diabetes duration, BP-lowering, lipid-lowering and diabetic treatment. The two subgroups did not differ for glycemic control, TG, HDL.

Within nonT2DM subjects, those with CV events had lower LV systolic longitudinal velocity $\mathrm{s}^{\prime}(8.0 \pm 1.2 \mathrm{vs}$ $9.3 \pm 1.2 \mathrm{~cm} / \mathrm{s} ; P=0.001)$ as compared to those without events, after adjustment for sex, age, BP, smoking habit, BP-lowering and lipid-lowering treatment.

\section{Discussion}

The present cross-sectional study investigated the relationships between plasma lipids and inflammatory cytokines as well as the impact of lipids and ILs on CV system in T2DM patients. Plasma ILs were directly related to plasma levels of MMP-12 that were inversely related to HDL. MMP-12 was independently associated with carotid diameter, arterial stiffness and LV mass, whereas HDL was independently associated with carotid wall thickness and LV diastolic longitudinal performance.
MMP-12 is a potent elastase highly expressed in macrophages [27]. Diabetes-related metabolic abnormalities, like hyperglycemia, promote macrophage activation and enhances the expression of the inflammatory mediators [28]. Inflammatory cytokines induce MMPs transcription and activation resulting in extracellular matrix alteration $[7,17,29]$ that, in the case of MMP-12 activation, is a cleavage of elastin. Elastin is the principal stress-bearing element of large arteries and its fragmentation leads to arterial dilation since arteries cannot withstand anymore the outward forces exerted by arterial pressure [30]. Degradation of elastin contributes also to arterial stiffening. In our T2DM patients, MMP-12 increased with plasma levels of IL- 6 and IL-18 and was independently related to elastic artery diameter and stiffness and to LV mass. The association between MMP-12 and LV mass was probably mediated by arterial stiffness as suggested by studies in mice with elastin haploinsufficiency [31].

The role of MMP-12 in arterial wall enlargement and stiffening has been demonstrated in experimental and clinical studies. In MMP-12 knock-out mice, the growth of aortic diameter and the expression of MMP-12 in 

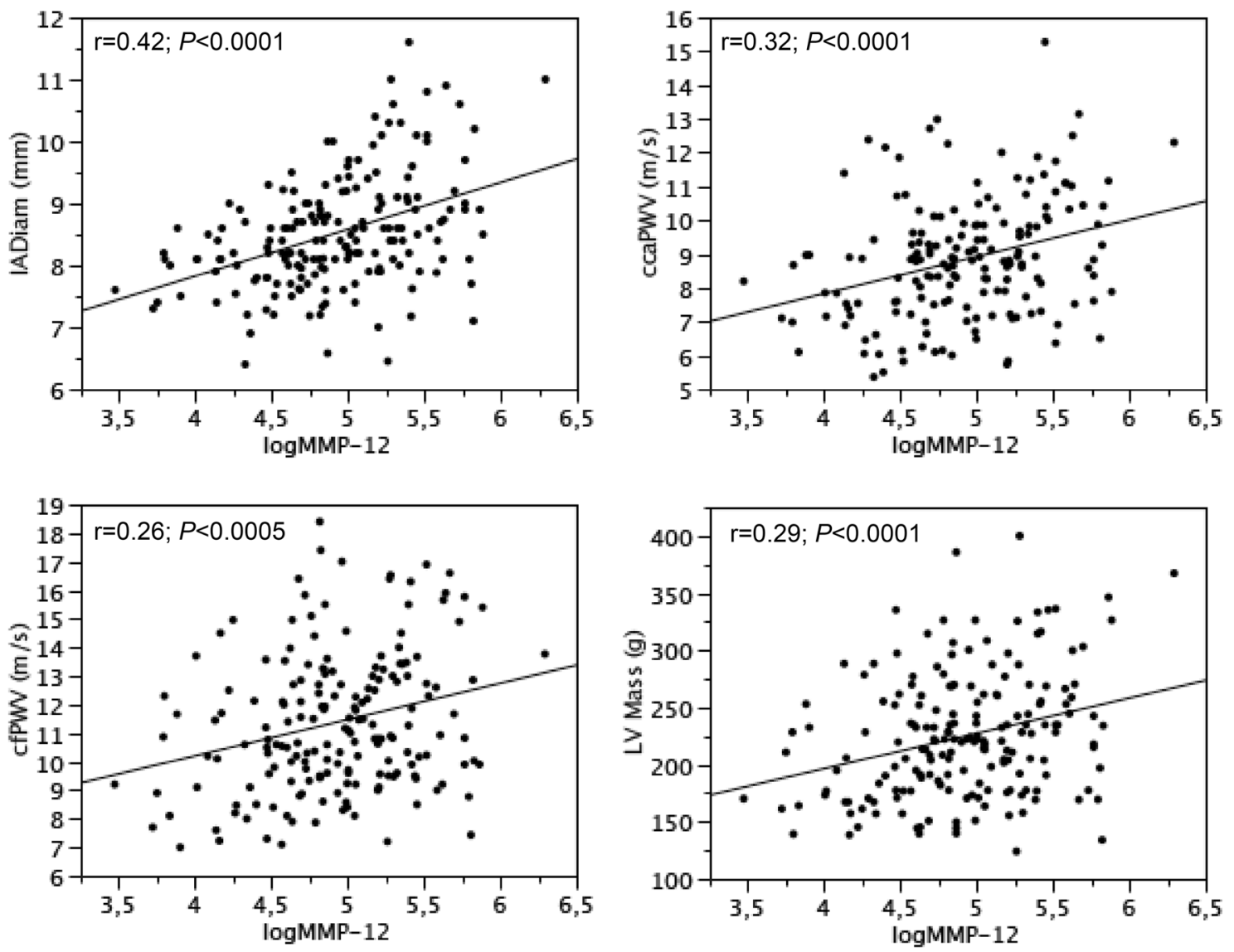

Fig. 3 Relationships between MMP-12 and carotid diameter, carotid wave speed, carotid-femoral pulse wave velocity and LV mass in T2DM patients

aortic wall were significantly lower as compared to wildtype mice [32], and an experimental injury of femoral artery did not induce increase in arterial stiffness and MMP-12 mRNA activation that were observed in wild-type mice [33]. In patients with acute dissection of ascending aorta, the plasma levels of MMP-12, IL-6, and IL-8 as well as MMP-12 activity in aortic wall were significantly higher as compared to subjects free of dissection $[34,35]$.

It is worth to note, that in nonT2DM subjects were observed the same direct associations between ILs and MMP-12 and between MMP-12 and CV measures as in T2DM patients. However, since the levels of ILs and MMP-12 were significantly lower, MMP-12 was independently related only to carotid diameter. This observation is in agreement with results of the Strong Heart Study showing that cardiac target organ damage precedes clinical appearance of T2DM and is related to inflammatory status [36].

Some members of the MMP family, including MMP12, can degrade the apolipoprotein A-I and decrease circulating HDL levels $[8,37]$. In T2DM and nonT2DM populations, plasma MMP-12 was inversely related to plasma HDL levels, which in turn were independently related to mitral longitudinal diastolic velocity $\mathrm{e}^{\prime}$ in both populations and to mitral longitudinal systolic velocity $s^{\prime}$ in nonT2DM subjects. Longitudinal velocities of mitral annulus provide important physiologic information on LV diastolic and systolic performance [25]. In experimental study on anesthetized dogs, an increase in early diastolic velocity $\mathrm{e}^{\prime}$ during dobutamine infusion was associated with reduction of $\tau$, consistent with faster relaxation, while the reduction in $\mathrm{e}^{\prime}$ velocity during ischemia was associated with prolongation of $\mathrm{\tau}$, consistent with slower relaxation [38]. Similarly, systolic s' velocity increased during dobutamine infusion in dosedependent manner and was strongly associated with $\mathrm{dP} / \mathrm{d} t_{\text {peak }}$ [39]. A direct association between HDL levels and LV longitudinal systolic and diastolic performance observed in our populations can be explained by studies showing that HDL increases insulin-independent glucose uptake in rat cardiomyocytes via an Akt signaling pathway [40] and that HDL mimetic peptide improves LV diastolic dysfunction in cholesterol-fed rabbits [41]. 

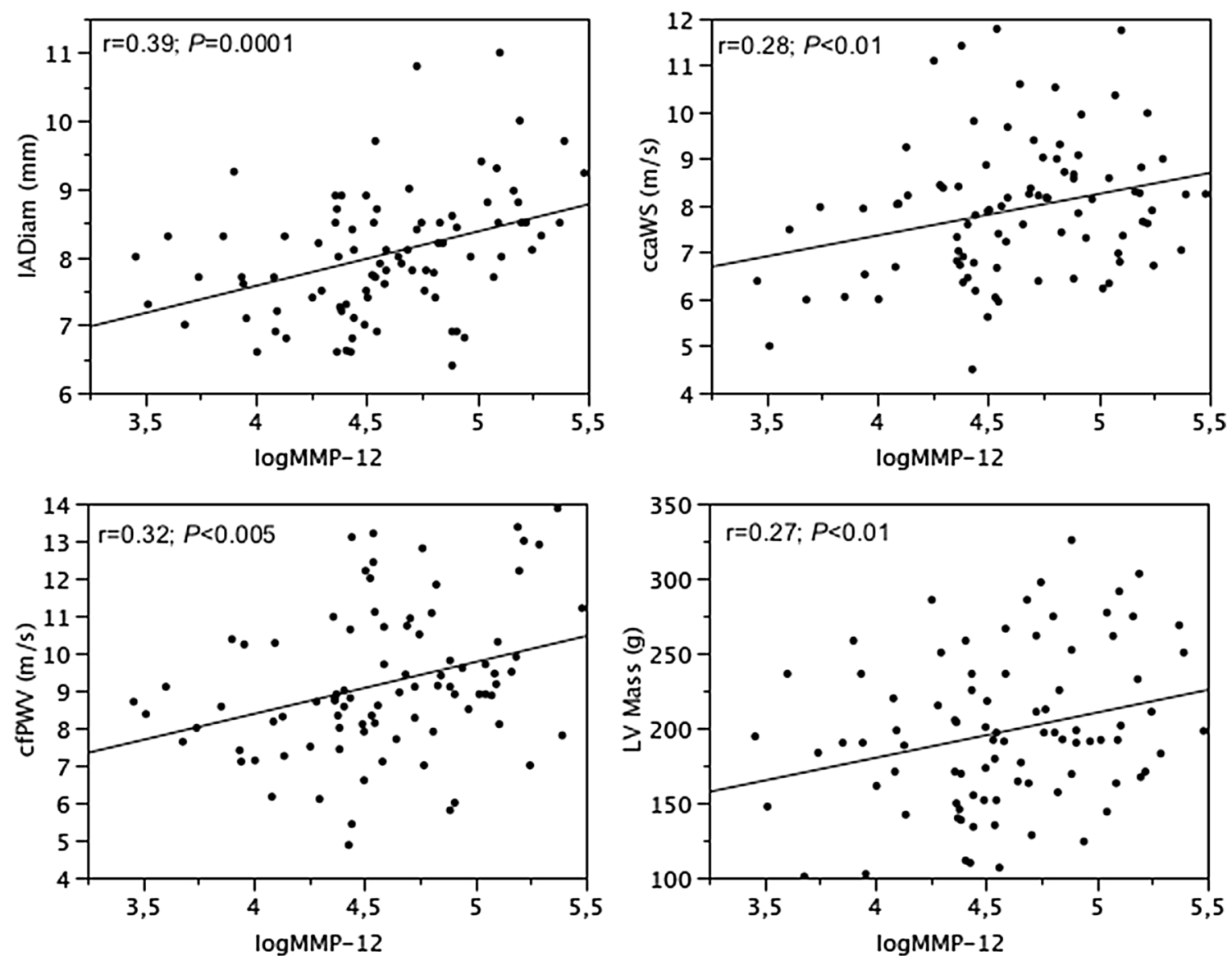

Fig. 4 Relationships between MMP-12 and carotid diameter, carotid wave speed, carotid-femoral pulse wave velocity and LV mass in nonT2DM subjects

Table 2 Determinants of vascular biomarkers in T2DM patients and in nonT2DM subjects

\begin{tabular}{|c|c|c|c|c|c|c|c|c|}
\hline & \multicolumn{2}{|l|}{ cIMT $(\mu \mathrm{m})$} & \multicolumn{2}{|l|}{ IAD (mm) } & \multicolumn{2}{|l|}{ ccaWS (m/s) } & \multicolumn{2}{|c|}{ cfPWV (m/s) } \\
\hline & T2DM & nonT2DM & T2DM & nonT2DM & T2DM & nonT2DM & T2DM & nonT2DM \\
\hline & $\beta \pm S E$ & & $\beta \pm S E$ & & $\beta \pm S E$ & & $\beta \pm S E$ & \\
\hline Sex (male) & & $0.20 \pm 0.09$ & $0.17 \pm 0.07$ & $0.37 \pm 0.09$ & & & & \\
\hline Age (years) & $0.22 \pm 0.07$ & $0.46 \pm 0.09$ & & & & $0.32 \pm 0.09$ & & $0.58 \pm 0.07$ \\
\hline Systolic BP (mmHg) & $0.15 \pm 0.06$ & & $0.17 \pm 0.06$ & & $0.26 \pm 0.06$ & $0.40 \pm 0.09$ & $0.30 \pm 0.06$ & $0.25 \pm 0.07$ \\
\hline $\mathrm{HDL}(\mathrm{mmol} / \mathrm{L})$ & $-0.33 \pm 0.07$ & & & & & & & \\
\hline BP therapy (yes) & & & & $0.20 \pm 0.09$ & & & $0.24 \pm 0.07$ & \\
\hline logMMP-12 & & & $0.36 \pm 0.06$ & $0.29 \pm 0.09$ & $0.27 \pm 0.06$ & & $0.17 \pm 0.06$ & \\
\hline$R^{2}$ & 0.17 & 0.25 & 0.22 & 0.32 & 0.18 & 0.26 & 0.22 & 0.48 \\
\hline$P$ & $<0.0001$ & $<0.0001$ & $<0.0001$ & $<0.0001$ & $<0.0001$ & $<0.0001$ & $<0.0001$ & $<0.0001$ \\
\hline
\end{tabular}

In T2DM patients, HDL was also independently and inversely related to cIMT. This association has been already described in a large meta-analysis including 21.000 subjects [14] and is probably related to the fact that HDL is able to suppresses smooth muscle cells migration and proliferation [42].

The combined role of ILs, MMP-12 and HDL in CV organ damage of T2DM patients was confirmed also by our observation that diabetic patients with established 
Table 3 Determinants of cardiac biomarkers in T2DM patients and in nonT2DM subjects

\begin{tabular}{|c|c|c|c|c|c|c|}
\hline & \multicolumn{2}{|l|}{ LV mass $(g)$} & \multicolumn{2}{|c|}{$\mathrm{s}^{\prime}$ velocity $(\mathrm{cm} / \mathrm{s})$} & \multicolumn{2}{|c|}{$\mathrm{e}^{\prime}$ velocity $(\mathrm{cm} / \mathrm{s})$} \\
\hline & T2DM & nonT2DM & T2DM & nonT2DM & T2DM & nonT2DM \\
\hline & $\beta \pm S E$ & & $\beta \pm S E$ & & $\beta \pm S E$ & \\
\hline Sex (male) & & $0.31 \pm 0.10$ & & & $0.26 \pm 0.08$ & \\
\hline Age (years) & & & & & $-0.22 \pm 0.07$ & $-0.27 \pm 0.09$ \\
\hline Body weight (kg) & $0.27 \pm 0.06$ & $0.32 \pm 0.09$ & $0.26 \pm 0.07$ & & & \\
\hline Systolic BP (mmHg) & $0.20 \pm 0.06$ & & & & & \\
\hline Heart rate (bpm) & & & $0.14 \pm 0.06$ & $0.22 \pm 0.09$ & & \\
\hline $\mathrm{HDL}(\mathrm{mmol} / \mathrm{L})$ & & & & $0.24 \pm 0.09$ & $0.33 \pm 0.07$ & $0.32 \pm 0.08$ \\
\hline logT2DM duration & $0.14 \pm 0.06$ & & & & & \\
\hline Stroke volume $(\mathrm{mL})$ & $0.41 \pm 0.06$ & $0.21 \pm 0.09$ & & & & \\
\hline LV mass $(\mathrm{g})$ & & & $-0.24 \pm 0.07$ & & $-0.17 \pm 0.07$ & \\
\hline LV wall stress $\left({ }^{*} 10^{3}\right.$ dynes $\left./ \mathrm{cm}^{2}\right)$ & & & $-0.20 \pm 0.07$ & & & \\
\hline logMMP-12 & $0.26 \pm 0.05$ & & & & & \\
\hline$R^{2}$ & 0.41 & 0.43 & 0.19 & 0.11 & 0.18 & 0.21 \\
\hline$P$ & $<0.0001$ & $<0.0001$ & $<0.0001$ & $<0.005$ & $<0.0001$ & $<0.0001$ \\
\hline
\end{tabular}

Table 4 Differences in lipids, ILs and MMP-12 between T2DM patients with and without CV organ damage and differences in ILs and CV measures in T2DM patients with and without CV disease

\begin{tabular}{|c|c|c|c|}
\hline $\mathrm{cIMT}(\mu \mathrm{m})$ & $>90$ th percentile (64) & Within limits (127) & $P$ \\
\hline $\mathrm{HDL}(\mathrm{mmol} / \mathrm{L})$ & $1.19 \pm 0.25$ & $1.31 \pm 0.30$ & $<0.005$ \\
\hline IL-18 (AU) & 1499 [790] & $1269[582]$ & 0.01 \\
\hline cfPWV $(\mathrm{m} / \mathrm{s})$ & $>$ 90th percentile (52) & Within limits (139) & $P$ \\
\hline $\mathrm{HDL}$ & $1.13 \pm 0.29$ & $1.32 \pm 0.27$ & $<0.0001$ \\
\hline MMP-12 (AU) & 158 [100] & $128[89]$ & $<0.05$ \\
\hline LVH & Yes (99) & No (92) & $P$ \\
\hline IL-6 & $38[28]$ & $34[23]$ & $<0.05$ \\
\hline MMP-12 (AU) & $155[112]$ & $124[86]$ & 0.01 \\
\hline CV disease & Yes (97) & No (94) & $P$ \\
\hline IL-6 (AU) & $39[28]$ & $32[22]$ & 0.01 \\
\hline $\operatorname{cfPWV}(\mathrm{m} / \mathrm{s})$ & $12.1 \pm 2.6$ & $10.7 \pm 2.1$ & $<0.05$ \\
\hline
\end{tabular}

organ damage, i.e. with increased carotid IMT, aortic stiffness and LV hypertrophy, had significantly lower plasma levels of HDL and higher levels of ILs and MMP12 as compared to those without vascular and cardiac impairment.

The only CV measure associated with the presence of $\mathrm{CV}$ disease in T2DM patients was cfPWV [43]. Indeed, increase in aortic stiffness has a wide impact on CV system. First, it reduces a cushioning function of large arteries thus increasing flow pulsatility in sensitive organs like kidney and brain $[44,45]$. Second, it anticipates a return of reflected waves to the heart in late systole, thus augmenting the late-systolic LV wall stress. Increase in LV wall stress stimulates adaptive thickening of myocardial fibers and development of LV hypertrophy [46, 47] that may negatively influence LV myocardial performance. In our T2DM patients, with increasing cfPWV increased end-systolic LV stress and LV mass, which both were inversely related to LV longitudinal systolic performance [48]. Interestingly, in nonT2DM subjects, the only CV parameter associated with CV events was LV longitudinal systolic velocity s' ${ }^{\prime}$, a finding consistent with results of meta-analysis demonstrating that global LV longitudinal strain is an accurate predictor of major adverse cardiac events [49]. 


\section{Study limitations}

The present study has some potential limitations. First, plasma insulin levels were not measured, and thus the impact of insulin on ILs, MMP-12 and CV biomarkers cannot be evaluated. Second, measuring HDL levels does not accurately depict their composition and functionality, which can be modified in diabetes. Third, LV diastolic performance was evaluated only by early diastolic longitudinal velocity of mitral annulus and no additional echocardiographic parameters related to LV relaxation and compliance were assessed. Finally, we are aware that cross-sectional design of the study cannot clearly elucidate cause-and-effect relationships.

\section{Conclusions}

Results of this cross-sectional study indicate that IL-6 and IL-18 trigger an increase in MMP-12 plasma levels, which in turn may reduce HDL levels and directly modify vascular structure and function, both in T2DM patients and nonT2DM subjects. Alterations in vascular structure and function elicit changes in the heart (Additional file 1: Figure S1). As expected, the levels of inflammatory cytokines, and consequently those of MMP-12, were significantly higher in T2DM patients that showed more prominent impairment of CV structure and function than nonT2DM subjects. Therefore, targeting inflammatory and MMPs' pathways could be a part of the strategy to prevent and control diabetesrelated CV complications $[11,50]$.

\section{Additional file}

Additional file 1: Figure S1. Role of inflammation and dyslipidemia in cardiovascular organ damage of T2DM patients.

\begin{abstract}
Abbreviations
T2DM: type 2 diabetes mellitus; CV: cardiovascular; TG: triglycerides; HDL: high density lipoprotein cholesterol; IL: interleukin; MMP: matrix-metalloproteinase; cfPWV: carotid-femoral pulse-wave velocity; CIMT: carotid intima-media thickness; IADiam: inter-adventitial diameter; ccaWS: local carotid wave speed; LV: left ventricle; s': systolic longitudinal velocity of mitral annulus; $e^{\prime}$ : early diastolic longitudinal velocity of mitral annulus.
\end{abstract}

\section{Acknowledgements}

Not applicable.

\section{Authors' contributions}

MK study conception, data analysis, paper writing. CM, IG data acquisition, data analysis, revision of the manuscript. AN, JN data analysis, revision of the manuscript. CP study conception, data analysis, revision of the manuscript. All authors read and approved the final manuscript.

\section{Funding}

Not applicable.

\section{Availability of data and materials}

The datasets used and/or analysed during the current study are not publicly available but are available from the corresponding author on reasonable request.

\section{Ethics approval and consent to participate}

The protocol of the study was approved by the ethics committee "Comitato Etico Area Vasta Nord-Ovest" (Reference Number: 3146/2010).

\section{Consent for publication}

Not applicable.

\section{Competing interests}

M Kozakova is responsible for Clinical Studies in Esaote, SpA, Genova, Italy.

\section{Author details}

${ }^{1}$ Department of Clinical and Experimental Medicine, University of Pisa, Via Savi 10, 56126 Pisa, Italy. ${ }^{2}$ Department of Surgical, Medical Molecular Pathology and Critical Care Medicine, University of Pisa, Via Savi 10, 56126 Pisa, Italy. ${ }^{3}$ Department of Clinical Sciences Malmö, Lund University, Jan Waldenströms gata 35, 20502 Malmö, Sweden.

Received: 25 January 2019 Accepted: 2 May 2019

Published online: 10 May 2019

\section{References}

1. Emerging Risk Factors Collaboration, Sarwar N, Gao P, Seshasai SR, Gobin $R$, Kaptoge S, et al. Diabetes mellitus, fasting blood glucose concentration, and risk of vascular disease: a collaborative meta-analysis of 102 prospective studies. Lancet. 2010;375:2215-22.

2. Parhofer KG. Interaction between glucose and lipid metabolism: more than diabetic dyslipidemia. Diabetes Metab J. 2015;39:353-62.

3. Bitzur R, Cohen H, Kamari Y, Shaish A, Harats B. Triglycerides and HDL cholesterol. Stars or second lead in diabetes? Diabetes Care. 2009;23(suppl 2):S373-7.

4. Wang X, Bao W, Liu J, Ouyang YY, Wang D, et al. Inflammatory markers and risk of type 2 diabetes: a systematic review and meta-analysis. Diabetes Care. 2013;36:166-75.

5. Zuliani G, Volpato S, Blè A, Bandinelli S, Corsi AM, et al. High interleukin-6 plasma levels are associated with low HDL-C levels in community-dwelling older adults: the InChianti study. Atherosclerosis. 2007;192:384-90.

6. Nonogaki K, Fuller GM, Fuentes NL, Moser AH, Staprans I. Interleukin-6 stimulates hepatic triglyceride secretion in rats. Endocrinology. 1995;136:2143-9.

7. Kossakowska AE, Edwards DR, Prusinkiewicz C, Zhang MC, Guo D, Urbanski SJ, et al. Interleukin-6 regulation of matrix metalloproteinase (MMP-2 and MMP-9) and tissue inhibitor of metalloproteinase (TIMP-1) expression in malignant non-Hodgkin's lymphomas. Blood. 1999;94:2080-9.

8. Lindstedt L, Saarinen J, Kalkkinen N, Welgus H, Kovanen PT. Matrix metalloproteinases-3, -7 , and -12 , but not -9 , reduce high density lipoproteininduced cholesterol efflux from human macrophage foam cells by truncation of the carboxyl terminus of apolipoprotein A-I. Parallel losses of pre-beta particles and the high affinity component of efflux. J Biol Chem. 1999;274:22627-34.

9. Goncalves I, Bengtsson E, Colhoun HM, Shore AC, Palombo C, SUMMIT Consortium, et al. Elevated plasma levels of MMP-12 are associated with atherosclerotic burden and symptomatic cardiovascular disease in subjects with type 2 diabetes. Arterioscler Thromb Vasc Biol. 2015;35:1723-31.

10. Mahmoud F, Al-Ozairi E. Inflammatory cytokines and the risk of cardiovascular complications in type 2 diabetes. Dis Markers. 2013;35:235-41.

11. Pollack RM, Donath MY, LeRoith D, Leibowitz G. Anti-inflammatory agents in the treatment of diabetes and its vascular complications. Diabetes Care. 2016;39(Suppl 2):S244-52.

12. Diamant M, Lamb HJ, van de Ree MA, Endert EL, Groeneveld Y, et al. The association between abdominal visceral fat and carotid stiffness is mediated by circulating inflammatory markers in uncomplicated type 2 diabetes. J Clin Endocrinol Metab. 2005;90:1495-501. 
13. Meléndez GC, McLarty JL, Levick SP, Du Y, Janicki JS, et al. Interleukin 6 mediates myocardial fibrosis, concentric hypertrophy, and diastolic dysfunction in rats. Hypertension. 2010;56:225-31.

14. Touboul PJ, Labreuche J, Bruckert E, Schargrodsky H, Prati P, et al. HDL-C, triglycerides and carotid IMT: a meta-analysis of 21,000 patients with automated edge detection IMT measurement. Atherosclerosis. 2014:232:65-71

15. Di Bonito P, Moio N, Scilla C, Cavuto L, Sibilio G, et al. Usefulness of the high triglyceride-to-HDL cholesterol ratio to identify cardiometabolic risk factors and preclinical signs of organ damage in outpatient children. Diabetes Care. 2012;35:158-62.

16. Wang X, Ye P, Cao R, Yang X, Xiao W, et al. Triglycerides are a predictive factor for arterial stiffness: a community-based 4.8-year prospective study. Lipids Health Dis. 2016;15:97.

17. Hobeika MJ, Thompson RW, Muhs BE, Brooks PC, Gagne PJ. Matrix metalloproteinases in peripheral vascular disease. J Vasc Surg. 2007:45:849-57.

18. American Diabetes Association. 2. Classification and diagnosis of diabetes. Diabetes Care. 2016;39(Suppt 1):S13-22.

19. Mattace-Raso F, Hofman A, Verwoert GC, Wittemana JC, Wilkinson I, et al. Determinants of pulse wave velocity in healthy people and in the presence of cardiovascular risk factors: 'establishing normal and reference values'. Eur Heart J. 2010;31:2338-50.

20. Kozakova M, Boutouyrie P, Morizzo C, Latta DD, Jamagidze G, et al. Radiofrequency-based wall tracking for noninvasive assessment of local carotid pulse pressure: comparison with applanation tonometry and association with organ damage. J Hypertens. 2018;36:2362-8.

21. Engelen L, Ferreira I, Stehouwer CD, Boutouyrie P, Laurent S, Reference Values for Arterial Measurements Collaboration. Reference intervals for common carotid intima-media thickness measured with echotracking: relation with risk factors. Eur Heart J. 2013;34:2368-80.

22. Marwick TH, Gillebert TC, Aurigemma G, Chirinos J, Derumeaux G, et al. Recommendations on the use of echocardiography in adult hypertension: a report from the European Association of Cardiovascular Imaging (EACVI) and the American Society of Echocardiography (ASE). Eur Heart J Cardiovasc Imaging. 2015:16:577-605.

23. de Simone G, Devereux RB, Daniels SR, Koren MJ, Meyer RA, Laragh JH. Effect of growth on variability of left ventricular mass: assessment of allometric signals in adults and children and their capacity to predict cardiovascular risk. J Am Coll Cardiol. 1995;25:1056-62.

24. Reichek N, Wilson J, St John Sutton M, Plappert TA, Goldberg S, et al. Noninvasive determination of left ventricular end-systolic stress: validation of the method and initial application. Circulation. 1982;65:99-108.

25. Silbiger JJ. Pathophysiology and echocardiographic diagnosis of left ventricular diastolic dysfunction. J Am Soc Echocardiogr. 2019;32:216-32.

26. Shore AC, Colhoun HM, Natali A, Palombo C, Khan F, SUMMIT Consortium, et al. Use of vascular assessments and novel biomarkers to predict cardiovascular events in type 2 diabetes: the SUMMIT VIP study. Diabetes Care. 2018:41:2212-9

27. Van Doren SR. Matrix metalloproteinase interactions with collagen and elastin. Matrix Biol. 2015;44-46:224-31.

28. Ahmed M, de Winther MPJ, Van den Bossche J. Epigenetic mechanisms of macrophage activation in type 2 diabetes. Immunobiology. 2017:222:937-43.

29. Raffetto JD, Khalil RA. Matrix metalloproteinases and their inhibitors in vascular remodeling and vascular disease. Biochem Pharmacol. 2008;75:346-59.

30. Duca L, Blaise S, Romier B, Laffargue M, Gayral S, et al. Matrix ageing and vascular impacts: focus on elastin fragmentation. Cardiovasc Res. 2016;110:298-308.

31. Faury G, Pezet M, Knutsen RH, Boyle WA, Heximer SP, et al. Developmental adaptation of the mouse cardiovascular system to elastin haploinsufficiency. Clin Invest. 2003;112:1419-28.

32. Longo GM, Buda SJ, Fiotta N, Xiong W, Greiner T, et al. MMP-12 has a role in abdominal aortic aneurysms in mice. Surgery. 2005:137:457-62.
33. Liu S-H, Bae YH, Yu C, Monslow J, Hawthorne EA, et al. Matrix metalloproteinase- 12 is an essential mediator of acute and chronic arterial stiffening Sci Rep. 2015;5:17189.

34. Proietta M, Tritapepe L, Cifani N, Ferri L, Taurino M, et al. MMP-12 as a new marker of Stanford-A acute aortic dissection. Ann Med. 2014;46:44-8.

35. Song Y, Xie Y, Liu F, Zhao C, Yu R, et al. Expression of matrix metalloproteinase-12 in aortic dissection. BMC Cardiovasc Disord. 2013;13:34.

36. de Simone G, Wang W, Best LG, Yeh F, Izzo R, et al. Target organ damage and incident type 2 diabetes mellitus: the Strong Heart Study. Cardiovasc Diabetol. 2017;16:64.

37. Noji Y, Kajinami K, Kawashiri MA, Todo Y, Horita T, Nohara A, et al. Circulating matrix metalloproteinases and their inhibitors in premature coronary atherosclerosis. Clin Chem Lab Med. 2001;39:380-4.

38. Opdahl A, Remme EW, Helle-Valle T, Lyseggen E, Vartdal T, Pettersen E, et al. Determinants of left ventricular early-diastolic lengthening velocity: independent contributions from left ventricular relaxation, restoring forces, and lengthening load. Circulation. 2009;119:2578-86.

39. Seo JS, Kim DH, Kim WJ, Song JM, Kang DH, Song JK. Peak systolic velocity of mitral annular longitudinal movement measured by pulsed tissue Doppler imaging as an index of global left ventricular contractility. Am J Physiol Heart Circ Physiol. 2010;298(5):H1608-15.

40. Heywood SE, Richart AL, Henstridge DC, Alt K, Kiriazis H, et al. Highdensity lipoprotein delivered after myocardial infarction increases cardiac glucose uptake and function in mice. Sci Transl Med. 2017;9:411.

41. Merlet N, Busseuil D, Mihalache-Avram T, Mecteau M, Shi Y, et al. HDL mimetic peptide CER-522 treatment regresses left ventricular diastolic dysfunction in cholesterol-fed rabbits. Int J Cardiol. 2016:215:364-71.

42. Tamama K, Tomura H, Sato K, Malchinkhuu E, Damirin A, et al. Highdensity lipoprotein inhibits migration of vascular smooth muscle cells through its sphingosine 1-phosphate component. Atherosclerosis. 2005:178:19-25.

43. Mansour AS, Yannoutsos A, Majahalme N, Agnoletti D, Safar ME, Ouerdane $\mathrm{S}$, et al. Aortic stiffness and cardiovascular risk in type 2 diabetes. J Hypertens. 2013;31:1584-92.

44. Verhave JC, Fesler P, du Cailar G, Ribstein J, Safar ME, Mimran A. Elevated pulse pressure is associated with low renal function in elderly patients with isolated systolic hypertension. Hypertension. 2005;45:586-91.

45. Okada K, Iso H, Cui R, Inoue M, Tsugane S. Pulse pressure is an independent risk factor for stroke among middle-aged Japanese with normal systolic blood pressure: the JPHC study. J Hypertens. 2011;29:319-24.

46. Ohyama Y, Ambale-Venkatesh B, Noda C, Chugh AR, Teixido-Tura G, et al. Association of aortic stiffness with left ventricular remodeling and reduced left ventricular function measured by MRI: the Multi-Ethnic Study of Atherosclerosis (MESA). Circ Cardiovasc Imaging. 2016;9:7.

47. Grossman W, Jones D, McLaurin LP. Wall stress and patterns of hypertrophy in the human left ventricle. J Clin Invest. 1975;56:56-64.

48. Dinh W, NickI W, Smettan J, Kramer F, Krahn T, et al. Reduced global longitudinal strain in association to increased left ventricular mass in patients with aortic valve stenosis and normal ejection fraction: a hybrid study combining echocardiography and magnetic resonance imaging. Cardiovasc Ultrasound. 2010:8:29.

49. Kalam K, Otahal P, Marwick TH. Prognostic implications of global LV dysfunction: a systematic review and meta-analysis of global longitudinal strain and ejection fraction. Heart. 2014;100:1673-80.

50. Kadoglou NP, Daskalopoulou SS, Perrea D, Liapis CD. Matrix metalloproteinases and diabetic vascular complications. Angiology. 2005;56:173-89.

\section{Publisher's Note}

Springer Nature remains neutral with regard to jurisdictional claims in published maps and institutional affiliations. 